\title{
Kutane Arthropodenreaktion nach Blutegeltherapie
}

\begin{abstract}
Zusammenfassung. Bei einer 69-jährigen Patientin wurden während eines stationären Aufenthaltes Blutegel im Bereich der unteren Lendenwirbelsäule bei Polyarthralgie angesetzt. Monate später führten ambulant durchgeführte Massagen unter Zuhilfenahme von ätherischen Ölsalben zu starkem Pruritus im Bereich der Bissstellen. Ein Jahr später kam die Patientin erneut zur stationären Aufnahme wegen progredienter Arthralgien. Im Bereich der Blutegelbisse fanden sich 9 gut kirschkerngroße, livid-rote juckende Papeln mit zentraler Hypopigmentierung. Zunächst bestand der Verdacht auf hypertrophe Narben. Zum Ausschluss einer Sarkoidose wurde die übliche Diagnostik mit Probeexzision durchgeführt. Histologisch fand sich eine pseudolymphomatoide entzündliche Gewebsreaktion mit follikulärer Hyperplasie und Gewebseosinophilie, das Gesamtbild passend zu einer Arthropodenreaktion. Eine Arthropodenreaktion ist eine sehr seltene Komplikation nach Blutegeltherapie. Es ist davon auszugehen, dass die Anwendung der ätherischen Ölsalbe eine Aktivierung der Arthropodenreaktion verursachte.
\end{abstract}

Cutaneous Reaction of Arthropods after Leech Therapy. A female 69-year old polyarthralgia inpatient was treated with leeches in the lumbar vertebral region. After massage continued for several months, using ethereal oils, pruritus occurred in the region of the bites. One year later she seturned as an inpatient due to progressing arthralgia. In the region of the bites several small humps with a central pale area were found. Keloid was at first suspected. To exclude sarcoidosis an excision and usual diagnostics were performed. Sarcoidosis could be excluded. Histologically a pseudolymphomatous inflammatory infiltrate with follicular hyperplasia and associated eosinophils was seen which was interpreted as a reaction to arthropods. Reaction to arthropods is a very rare complication after leech therapy. It is speculated that the application of ethereal oils caused an activation of the reaction to arthropods.

Akt Dermatol 2001; 27: $42-46$

(c) Georg Thieme Verlag Stuttgart $\cdot$ New York ISSN 0340-2541

\author{
A.-M. Beer ${ }^{1}$, S. Fey ${ }^{1}$, C. Kuhnen², T. Mentzel ${ }^{3}$ \\ ${ }^{1}$ Modellabteilung für Naturheilkunde, Klinik Blankenstein, \\ Hattingen (Chefarzt: Dr. med. A.-M. Beer) \\ ${ }^{2}$ Institut für Pathologie an den Berufsgenossenschaftlichen \\ Kliniken Bergmannsheil-Universitätsklinik, Bochum \\ (Direktor: Prof. Dr. med. K.-M. Müller) \\ ${ }^{3}$ Dermatohistopathologische Gemeinschaftspraxis, \\ Friedrichshafen
}

\section{Einleitung}

Der medizinische Blutegel, Hirudo medicinale, gehört zur Gruppe der Ringelwürmer. Er ernährt sich von Blut, von Fischen, Fröschen oder Säugetieren. Im Schlund hat er drei sternförmig angeordnete Kiefer, so dass sein Biss die Form eines „Mercedes“-Sterns zeigt. Der Speichel des Blutegels enthält viele Wirkstoffe (Hirudin, Calin, Hyaluronidase, Egline, Bdellin, Apyrase, Kollagenase, Destabilase, Piyavit), die heute in synthetischer Form, vor allem in der plastischen Chirurgie Anwendung finden. In der Naturheilkunde werden Blutegel vor allem zur Behandlung von Erkrankungen verwendet, die mit einem unerwünschten Blutstau, Schwellungen und Ödemen einhergehen. Als Indikationen gelten die Krampfadern und sonstige Venenleiden, der akute Gichtanfall, die rheumatischen Beschwerden (Gelenkschmerzen), die Migräne, Nasennebenhöhlenentzündungen.

In der Abteilung Naturheilkunde der Klinik Blankenstein wird der Blutegel regelmäßig eingesetzt, da sein Biss entzündungshemmend wirkt, antibiotische Effekte zeigt und zur Schmerzlinderung beiträgt. In seltenen Fällen finden sich Reaktionen an den Bissstellen.

Eine sehr seltene Therapiekomplikation wird in der Kasuistik beschrieben.

\section{Kasuistik}

\section{Anamnese}

Bei einer 69-jährigen Patientin bestehen progrediente Polyarthralgien, insbesondere Gonarthralgien. 1998 war bereits eine perkutane Neurolyse des Ramus dorsalis im Bereich der LWS durchgeführt worden. Trotz intensiver ambulanter Maßnahmen (physikalische Therapie, Schmerzmedikation etc.) kam es zu keiner Besserung, so dass die Patientin 12/98 erstmals in die Abteilung Naturheilkunde eingewiesen wurde. Es erfolgte eine Blutegelbehandlung im Bereich der unteren LWS/Sakrumbereich (Fa. Altmann, Schorndorf). Die durchgeführte Blutegeltherapie führte zu erheblicher Besserung der Beschwerden. 9 Monate später wurden im ambulanten Bereich klassische Massagen im LWS-Bereich unter Zuhilfenahme ätherischer Ölsalben durchgeführt. Die Patientin berichtete, dass im Anschluss an die Massagen ein starker Juckreiz im Bereich der ehemaligen primär verheilten Bissstellen, an de- 
nen 9 Monate zuvor eine Blutegeltherapie durchgeführt worden war, auftrat. Es sei zu einer Knötchenbildung im Bereich der unteren Lendenwirbelsäule gekommen.

\section{Aufnahmebefund}

Im November 1999 wurde die Wiederaufnahme in die Abteilung Naturheilkunde der Klinik Blankenstein notwendig, da sich das Beschwerdebild erneut deutlich verschlechtert hatte. Es bestanden Schmerzen in der gesamten Schulterregion mit Ausstrahlung in beide Arme im Sinne einer Zervikobrachialgie. Rezidivierende Lumbalgien bei Facettensyndrom L4/5 - L5/S1 standen ebenso im Vordergrund wie die kirschkerngroßen Papeln im Bereich der unteren LWS/Sakrumbereich (Abb.1).

\section{Hautbefund}

Dermatologischerseits wurden 9 gruppiert stehende gut kirschkerngroße, livid-rote, juckende, ca. 2-3 mm über dem Hautniveau erhabene, glatt begrenzte Papeln mit zentraler Hypopigmentierung im Bereich der unteren Lendenwirbelsäule/ Sakrumbereich beschrieben. Verdachtsdiagnose: hypertrophe Narben. Zum Ausschluss einer Sarkoidose oder Fremdkörpergranulomen wurde eine weitere Diagnostik durchgeführt.

\section{Allgemeinbefund}

Abdomen, Cor, Pulmo unauffällig. Merieux-Stempeltest unauffällig. Lymphknoten nicht palpabel.

\section{Mikroskopische Befunde}

Es wurde eine Probeexzision aus den betroffenen Hautstellen entnommen. Feingeweblich Akanthose mit fokalen Serumeinschlüssen im oberen Stratum corneum. Diskrete Hyperkeratose. Leichte Exozytose. Epidermodermale Grenze scharf. Dichte lymphohistiozytäre Infiltration im Stratum reticulare, betont um Hautadnexstrukturen. Insgesamt keilförmiges entzündliches Infiltrat mit pseudolymphomatoider Struktur und deutlicher lymphofollikulärer Hyperplasie. Dichte Infiltrate eosinophiler Granulozyten. Keine atypischen Zellinfiltrate. Unter Berücksichtigung der eindeutigen klinischen Identifikation der Bissstellen an der Region der Gewebsentnahme konnte das morphologische Bild als eine Arthropodenreaktion der Haut mit pseudolymphomähnlichem Entzündungsinfiltrat mit lymphofollikulärer Hyperplasie eingeordnet werden (Abb. 2 u.3).

\section{Radiologische, klinisch-chemische Befunde}

Röntgen-Thorax: Mäßige Linksverbreiterung des Herzens ohne Lungenstauung, kein Anhalt für Hilus- oder Mediastinallymphom im Sinne einer Sarkoidose.

Laboruntersuchungen: Blutbild, Differenzialblutbild, Serumchemie, Gerinnung, BSG, CRP unauffällig.

Mikrobiologie der Faeces ebenfalls unauffällig.

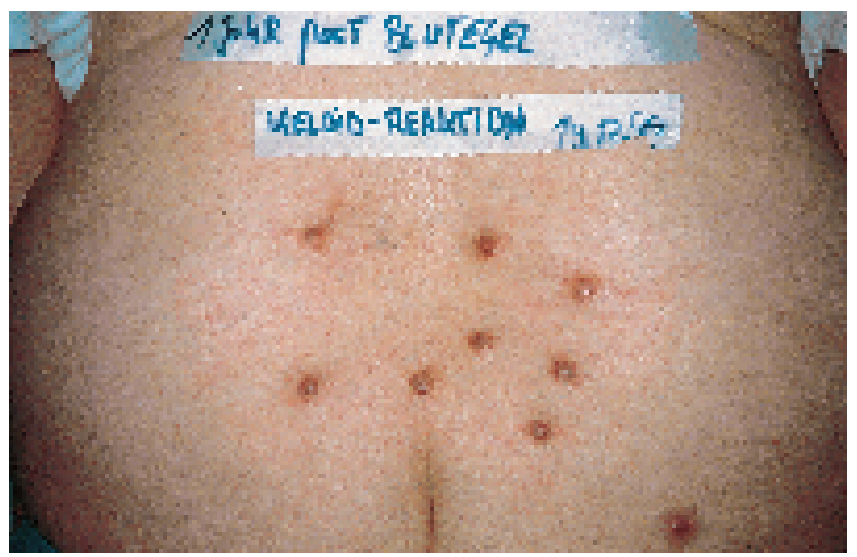

Abb.1 Papeln im Bereich der unteren LWS.

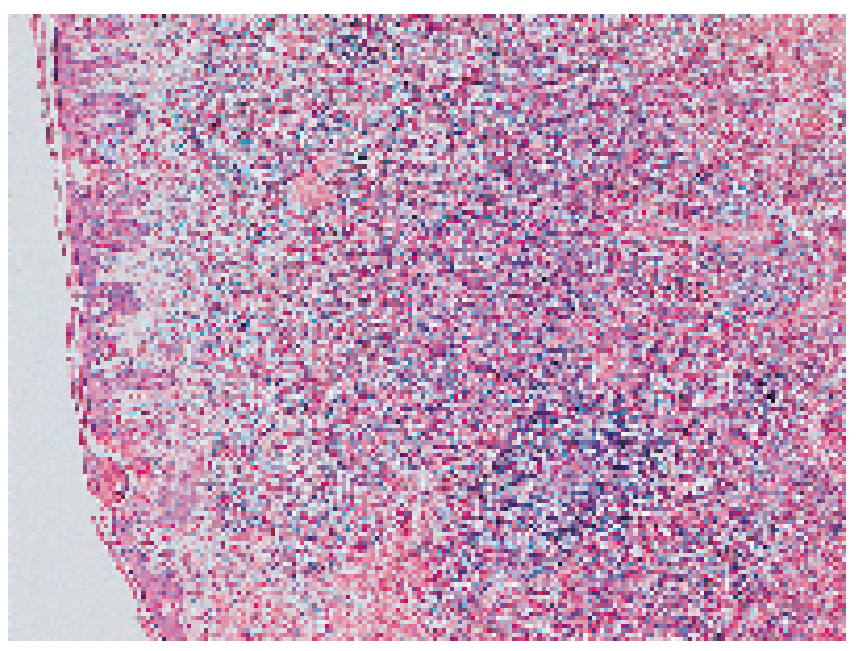

Abb. 2 Pseudolymphomatoide Entzündungsreaktion mit keilförmig zur Tiefe reichendem Entzündungsinfiltrat und lymphofollikulärer Hyperplasie $(\mathrm{HE} \times 2,5)$.

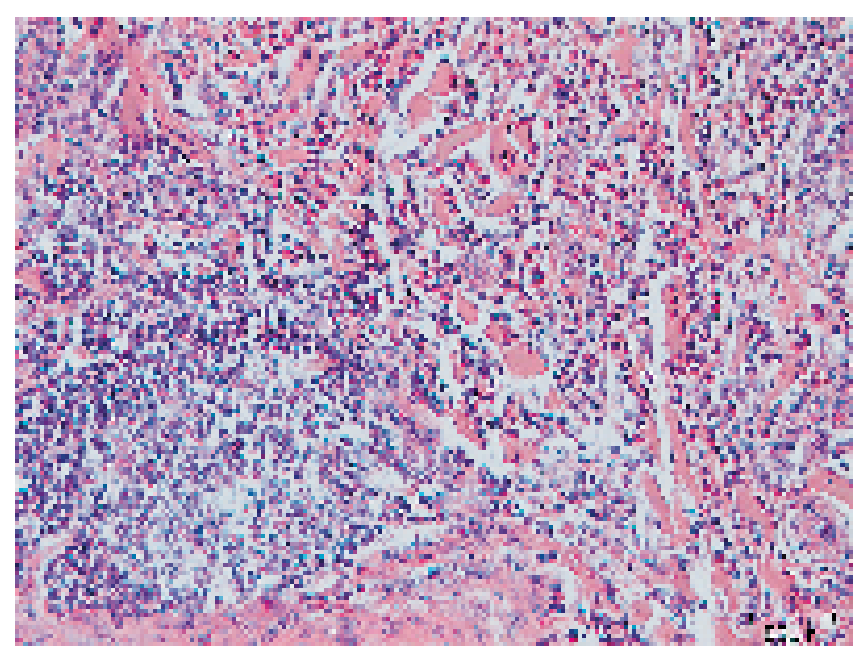

Abb. 3 Detailausschnitt: lymphomatoide Entzündung mit Endothelproliferaten und dicht gelagerten eosinophilen Granulozyten $(\mathrm{HE} \times 20)$. 


\section{Therapie und Verlauf}

Therapeutisch wurden bei der Patientin die klassischen Naturheilverfahren (Phyto-, Hydro-, Thermo-, Ernährungs-, Ordnungs- und Bewegungstherapie) angewendet. Die Symptomatik der Patientin besserte sich daraufhin. Auf Massagen im Bereich der unteren LWS wurde verzichtet. Lokal kamen Einreibungen mit Dimetindenmaleat im LWS-Bereich zur Anwendung.

\section{Diskussion}

Bei der Patientin traten 9 Monate nach Blutegeltherapie im LWS-/Sakrumbereich, im Anschluss an eine klassische Massage unter Zuhilfenahme ätherischer Ölsalben, an den zuvor abgeheilten Blutegel-Bissstellen stark juckende Hauteffloreszenzen auf. Bei der stationären Aufnahme wurde zunächst der klinische Verdacht auf hypertrophe Narben bzw. Fremdkörpergranulome geäußert. Eine Sarkoidose konnte mit den üblichen laborchemischen und radiologischen Maßnahmen ausgeschlossen werden. Zur eindeutigen diagnostischen Abklärung wurden Probeexzisionen entnommen.

In der Differenzialdiagnose der hier beschriebenen kutanen Arthropodenreaktion nach Blutegeltherapie, sind histologisch insbesondere lymphoproliferative Läsionen (sog. Pseudolymphome und Lymphome) und Prozesse mit Gewebseosinophilie zu berücksichtigen. Die lymphomatoide Papulose (Macaulay) zeigt feingeweblich ein keilförmiges Infiltrat aus atypischen Lymphozyten mit neutrophilen und eosinophilen Granulozyten sowie mononukleären Entzündungszellen. Die atypische lymphoide Zellpopulation besteht aus teils mehr pleomorphen (CD 30-positiven), teils mehr kleineren atypischen Lymphozyten. Klinisch treten rezidivierend erythematöse Papeln auf [1]. Kutane Infiltrate eines M. Hodgkin können ebenfalls einen erheblichen Anteil eosinophiler Granulozyten aufweisen, die Identifizierung der Hodgkin- und Sternberg-Reed-Zellen u.U. mit ergänzenden immunhistochemischen Methoden (CD 15, CD 30) erlauben die Einordnung bei einer Lymphogranulomatose Hodgkin. Eosinophile Granulozyten finden sich auch bei der Langerhans-Zellhistiozytose (Histiozytosis X), die vermehrt im Kindesalter kutane, makulopapulöse Läsionen zeigt. Histologisch sind in den Hautläsionen neben Eosinophilen die für die Erkrankung pathognomonischen atypischen Langerhans-Zellen mit kaffeebohneartigen Zellkernen (S-100- und CD 1 a-positiv) nachweisbar. Schließlich ist differenzialdiagnostisch die Scabies zu berücksichtigen; histologisch sind oft (ggf. erst in Stufenschnitten) Reste der Skabiesmilbe in intraepidermalen Gangstrukturen nachweisbar, eosinophile Granulozyten können massenhaft im oberen Korium und als keilförmiges Infiltrat zur Tiefe vorliegen.

Sämtliche hier aufgeführten Differenzialdiagnosen ließen sich insbesondere unter Berücksichtigung der eindeutigen klinischen Angaben zur Anamnese und zur Lokalisation der Läsion ausschließen. Unter Berücksichtigung der Klinik eindeutig identifizierter Bissstellen in dieser Hautregion ist das morphologische Bild als eine Arthropodenreaktion einzuordnen.

Es handelt sich um eine außerordentlich seltene Reaktion im Rahmen von Blutegeltherapien, die bislang in der Literatur nicht beschrieben ist. Im Regelfall treten $12-48 \mathrm{~h}$ nach Blutegeltherapie höchstens kosmetisch störende, rasch vorüberge- hende Hautveränderungen in Form von Mercedes-Stern-förmigen Bisswunden auf, deren Ränder anschwellen und im Umkreis Ekchymosen bis ca. 0,5 cm Durchmesser aufweisen. Die größte Belästigung für den Patienten geht von dem begleitenden Pruritus aus. Nach 8-10 Tagen heilen die Bisswunden mit kleinen Narben ab, die Umgebungsrötung verblasst vollkommen.

Die bisher in der Literatur veröffentlichten Komplikationen nach Blutegeltherapien mit dem in Europa und den Vereinigten Staaten am häufigsten verwendeten „Hirudo medicinalis“ berichten vor allem sowohl über das Auftreten von eng lokalisierten kleineren Wundkomplikationen, wie z.B. der Zellulitis, als auch ausgedehnteren Infektionen wie Erysipel oder Phlegmone, die besonders dann auftreten, wenn die Blutegel noch vor dem normalen Abfallen abgerissen oder wenn frühzeitig die Nachblutungen gestillt werden. Als Ursache der Infektionen werden insbesondere Aeromonas Species, an erster Stelle der im Darm von Blutegeln lebende Darmsymbiont „Aeromonas hydrophila“, ein gramnegatives Stäbchenbakterium, angesehen [2].

Nachdem die Blutegeltherapie in den achtziger Jahren in der plastischen und rekonstruktiven Chirurgie eine Renaissance besonders zur venösen Dekongestion von gestielten Lappen und mikrovaskulären freien Gewebetransplantaten erlebte, wird über Infektionsraten von 7-20\% berichtet. Tritt eine klinisch signifikante Infektion nach Blutegelbehandlung in Erscheinung, so sinkt die Erfolgsrate des Lappens von 70-80\% auf nur noch $30 \%$.

Aeromonas hydrophila kann sowohl große nekrotisierende Weichteil- und Muskelinfektionen, als auch Osteomyelitis und Septikämie hervorrufen [3].

Des Weiteren wird von Weichteilinfektionen mit „Aeromonas sobria“, „Aeromonas cavia“, „Serratia marcescens“ und „Enterobacter aerogenes“ nach Blutegeltherapie mit Hirudo medicinalis [4-8] berichtet sowie eine Infektion mit „Aeromonas hydrofila“ nach Anwendung des Blutegels Hirudo michaelseni [9].

Aufgrund des möglichen Infektionsrisikos mit dem Blutegelbazillus empfehlen viele Autoren eine prophylaktische Antibiotikatherapie mit Cefalosporinen der dritten Generation, Aminoglycosiden, Tetrazyklinen oder Cotrimoxazol. Aeromonas hydrophila zeigte sich resistent gegen Penicillin, Ampicillin und Cefalosporine der ersten Generation [10]. Auch die Übertragung von HIV-Viren durch Blutegel wird diskutiert, allerdings nur beim Baden in afrikanischen Seen. Bei Verwendung von Zuchtegeln und einmaligem Gebrauch besteht keine Übertragungsmöglichkeit [11].

Weitere Komplikationen nach Blutegeltherapie sind in den Bereich der allergischen Reaktionen einzuordnen. Es wird von Erythemen, Pruritus, Hämorrhagien und flüchtigen urtikariellen Erscheinungen als Wirkung des von den Blutegeln direkt abgegebenen Histamins bis hin zum anaphylaktischen Schock berichtet $[12,13]$.

Auch die Ausbildung von allergischen Kontaktdermatitiden im Sinne einer Typ-IV-Allergie auf den Blutegel-Wirkstoff Rohhirudin nach lokaler Salbenapplikation wird beschrieben. Der 
Wirkstoff kommt besonders bei der Behandlung von Hämorrhoidalleiden, Thrombophlebitiden und Sportverletzungen zur Anwendung [14,15].

Bislang werden in der Literatur überwiegend Komplikationen vorgestellt, die zeitlich in kürzerem Zusammenhang mit der Blutegelbehandlung stehen. An Spätkomplikationen wird lediglich über das Auftreten einer „Anetodermie“ mit atrophischen, hypochromen Hautveränderungen, 40 Jahre nach Applikation von Blutegeln, berichtet [16].

Empirisch kommen in der Abteilung Naturheilkunde der Klinik Blankenstein jährlich ca. 2000 Blutegelexemplare der Art Hirudo medicinalis im Rahmen der klassischen naturheilkundlichen Therapie zum Einsatz. Der oben beschriebene Kasus einer Hautspätreaktion in Form von pruriginösen Papeln 9 Monate post Blutegeltherapie trat erstmalig auf. Es ist davon auszugehen, dass die Massagetherapie unter Zuhilfenahme ätherischer Öle oder der mechanische Einfluss durch die Massage selbst eine Reaktion an den Bissstellen ausgelöst haben könnte. Diese Fragen ließen sich möglicherweise durch eine erneute Exposition der Patientin mit einem Blutegel und durch alleinige oder kombinierte Testung des ätherischen Massageöls klären.

\section{Literatur}

${ }^{1}$ Karp DL, Horn TD. Lymphomatoid papulosis. J Am Acad Dermatol 1994; 30: 379-395

${ }^{2}$ Müller IW. Handbuch der Blutegeltherapie: Theorie und Praxis. 1. Aufl. Heidelberg: Haug, 2000

${ }^{3}$ de Chalain TMB. Exploring the use of the medicinal leech: a clinical risk-benefit analysis. J Reconstr Micr Surg 1996; 12 (3): 165 172

${ }^{4}$ Fenollar F, Fournier PE, Legre R. Unusual case of Aeromonas sobria Cellulitis Associated with the Use of Leeches. Eur J Clin Microbiol Infect Dis 1999; 18 (1): $72-73$

${ }^{5}$ Mackay DR, Manders EK, Saggers GC, Banducci DR, Prinsloo J, Klugman K. Aeromonas Species Isolated from Medicinal Leeches. Ann Plast Surg 1999; 42 (3): 275-279

${ }^{6}$ Graf J. Symbiosis of Aeromonas veronii Biovar sobria and Hirudo medicinalis, the Medicinal Leech: a Novel Model for Digestive Tract Associations. Infect Immun 1999; 67 (1): 1 - 7

${ }^{7}$ Pereira JA, Greig JR, Liddy H, Ion L, Moss ALH. Leech-borne Serratia marcescens infection following complex hand injury. Br J Plast Surg 1998; 51: 640-664

${ }^{8}$ van Wingerden JJ, Oosthuizen JH. Use of the local leech Hirudo michaelseni in reconstructive plastic and hand surgery. S Afr J Surg 1997; 35 (1): 29-31

${ }^{9}$ Nonomura H, Kato N, Ohno Y, Itokazu M, Matsunaga T, Watanabe K. Indigenous bacterial flora of medicinal leeches and their susceptibilities to 15 antimicrobial agents. J Med Microbiol 1996; 45 (5): $490-493$

${ }^{10}$ Mehlhorn H. Übertragung von HIV durch Blutegel. Hautarzt 1993; $44: 744$

${ }^{11}$ Ross MS. The Leech: of Dermatologic Interest? Arch Dermatol 1983; 119: 276

12 Heldt TJ. Allergy to Leeches. Henry Ford Hosp Med Bull 1961; 9: $498-519$

${ }^{13}$ Gall H von, Frommer A. Kontaktallergie auf den Blutegel-Extrakt Rohhirudin. Derm Beruf Umwelt 1993; 41/2: 77 - 78

${ }^{14}$ Dejobert Y, Martin P, Thomas P, Bergoend H. Contact dermatitis from topical extract. Contact Derm 1991; 24 (5): 366 - 367
${ }^{15}$ Zollner TM, Gall H, Völpel H, Kaufmann R. Type IV allergy to natural hirudin confirmend by in vitro stimulation with recombinant hirudin. Contact Derm 1996; 35 (1): 59-60

${ }^{16}$ Siragusa M, Batolo D, Schepis C. Anetoderma Secondary to the Application of Leeches. Int J Dermatol 1996; 35: 226

Dr. med. André-Michael Beer

Modellabteilung für Naturheilkunde

Klinik Blankenstein

Im Vogelsang 5 - 11

45527 Hattingen 


\section{BUCHBESPRECHUNG}

Nanna Schürer, J. Kresken: Die trockene Haut. 2000. 103 Seiten, 43 Abb., 15 Tab. Wissenschaftliche Verlagsgesellschaft $\mathrm{mbH}$, Stuttgart. Kart. DM 38,-. ISBN 3-8047$1776-4$

Die Zusammenarbeit zwischen Dermatologen und Apothekern ist wichtig, wenn die Versorgungskette bei dermatologischen Patienten gelingen soll. Viele Patienten konsultieren wegen trivialer Hautprobleme den Apotheker, und in seiner Verantwortung liegt es, die ggf. notwendige dermatologische Betreuung zu induzieren und nicht zu verzögern. Umgekehrt ist der Dermatologe in der Rezeptur von Externa in besonderem Maße auf den Sachverstand und die galenische Erfahrung des Apothekers angewiesen. Insofern ist es erfreulich, dass Frau Privatdozentin Nanna Schürer, Dermatologin in Osnabrück, und Dr. Joachim Kresken, Apotheker und in der Gesellschaft für Dermopharmazie engagierter Brückenbauer zwischen Dermatologie und Pharmazie, in der wissenschaftlichen Verlagsgesellschaft Stuttgart ein Bändchen über „Die trockene Haut" herausgegeben haben. Das Buch stellt zunächst anatomische und biochemische Grundlagen der Hautbarriere dar, geht dann auf Dermatosen ein, die mit trockener Haut einhergehen, referiert berufliche und alltägliche Konsequenzen für Patienten mit trockener Haut, erläutert die medikamentöse Therapie und Prophylaxe und stellt schließlich kosmetische Maßnahmen bei trockener Haut dar. Leider wird nicht ganz deutlich, an wen sich das Buch richtet. Obwohl die Autoren im Vorwort zu Recht monieren, dass Kapitel über die trockene Haut in gängigen Lehrbüchern der Dermatologie fehlen, dürften die im vorliegenden Band dargestellten Erkenntnisse den Dermatologen bestens bekannt sein. Man muss daher davon ausgehen, dass das Buch eher für den Allgemeinarzt oder den Apotheker geschrieben wurde. Unter diesen Prämissen ist es akzeptabel, dass vieles lückenhaft bleibt. So ist beispielsweise unverständlich, dass im Rahmen des atopischen Ekzems lediglich auf die Kriterien nach Hanifin und Rajka hingewiesen wird, ohne die für die praktische Diagnostik viel wichtigeren „evidenzbasierten“ Erlanger Atopiektriterien zu erwähnen. Ebenso vermisst man bei der Darstellung des Exsikkationsekzems als klassische, exogen induzierte Xerosis cutis den Hinweis auf den häufigen Seifen- oder Syndetabusus. Die wenigen Zeilen über die beruflichen und alltäglichen Konsequenzen der trockenen Haut dürften auch kaum ausreichen, um beispielsweise Anwärter von Hautrisikoberufen zu beraten. Zusammenfassend bietet das Buch eine gute Einführung in die Problematik der trockenen Haut, wobei sich der Dermatologe freilich vertiefteren Darstellungen zuwenden wird. 